\title{
On the modification of the method of mechanical quadrature for SIE in crack problems under step-like loads
}

\author{
A. N. Galybin \\ The Schmidt Institute of Physics of the Earth RAN, Russia
}

\begin{abstract}
A number of recently published studies are devoted to modelling of hydraulic fracture propagation in elastic rocks (reservoirs). The modern approaches assume modelling of the hydro-fracture by an open crack (in general curvilinear) loaded by a normal pressure distributed over the central part of the crack which surfaces are stress-free near the crack ends; so-called lag. Such step-like loads generate certain errors in computations if the standard method of mechanical quadrature, MMQ, is used to solve the system of singular integral equations, SIE. To eliminate these errors we suggest an approach that is somewhat similar to the one suggested by Savruk in Two-dimensional problems of elasticity for crack problems (1981) for a crack under concentrated load. The solution is sought as the sum of two solutions. The first solution addresses the discontinuities in the right-hand side of the SIE and corresponds to the case of a rectilinear crack under step-like load. This solution is found in analytical form via singular integrals. The second solution is found numerically by the standard MMQ from the full SIE with a smooth right-hand side obtained after integration of the first solution multiplied by the regular part of the kernel. Therefore the accuracy of the total solution is controlled by its numerical (second) solution. The paper presents a benchmark solution for a SIE with a degenerated regular kernel and a numerical solution for two parallel cracks under step-like loads (as a model of multi-stage hydro-fracture).
\end{abstract}

Keywords: singular integral equations, discontinuous load, quadrature formulas, hydrofracture. 


\section{Introduction}

The crack problems in $2 \mathrm{D}$ can be reduced to a singular integral equation, SIE, of the following form, see e.g. Savruk [1]

$$
\frac{1}{\pi} \int_{-1}^{1}\left[\left(\frac{1}{t-x}+K(t, x)\right) g(t)+L(t, x) \overline{g(t)}\right] d t=p(x), \quad|x|<1
$$

where $g(t)$ is an unknown function proportional to the density of the crack opening displacements that satisfies the Holder condition; $K(t, x)$ and $L(t, x)$ are bounded kernels continuous in $(-1,1)(-1,1) ; p(x)$ is a known load on the crack.

The unknown function satisfies the following condition of single-valuedness of the displacements

$$
\int_{-1}^{1} g(t) d t=0
$$

The system (1)-(2) possesses a unique solution unbounded at both ends with the square root singularity.

For smooth loads applied on the whole surfaces of a crack the system (1)-(2) can be solved by using the Gauss-Chebyshev quadrature formulas by the method of mechanical quadrature, MMQ, as detailed in the next section. Its solution is stable with respect to small changes in the right hand side (loads). The accuracy of the numerical solution depends upon the number of nodes (denoted as $n$ further on) in the Gauss-Chebyshev quadrature formula for singular integrals, which is accurate for polynomials of the $(2 n-1)$ degree. The increase of $n$ in the quadrature formula (and the number of collocation points respectively) allows one to refine the solution to achieve given accuracy. However, for discontinuous step-like loads it is impractical to rich high accuracy due to limitations in computer recourses even for the case of several interacting cracks. This case however presents certain interest for practice, for example, in modelling of hydrofracture propagation where the loads with lag act on the crack surface, i.e. the crack is loaded in the middle by normal loads but it is stress-free near the ends.

The paper presents an approach to handle such loads by using the MMQ technique with insignificant increase of computer time required to make smooth the right-hand side of the SIE, which requires extra integration. Instead the accuracy of the solution can be dramatically improved without increasing the number of the nodes/collocation points, which allows one to consider a large number of cracks under step-like loads by using moderate computer resources. 


\section{Approach for discontinuous loads}

The standard numerical approach for solving the system (1)-(2) assumes the use of the Gauss-Chebyshev quadratures for singular integrals, which reduces the system to a system of linear algebraic equations, SLAE, of the following form

$$
\left\{\begin{array}{c}
\frac{1}{n} \sum_{k=1}^{n}\left[\left(\frac{1}{\xi_{k}-\eta_{j}}+K\left(\xi_{k}, \eta_{j}\right)\right) q_{k}+L\left(\xi_{k}, \eta_{j}\right) \bar{q}_{k}\right]=p\left(\eta_{j}\right) \\
\sum_{k=1}^{n} q_{k}=0
\end{array}\right.
$$

Here the following notations have been introduced

$$
q_{k}=g\left(\xi_{k}\right) \sqrt{1-\xi_{k}^{2}}
$$

for unknown values of the density of the crack opening displacements at the nodes of the Gauss-Chebyshev quadrature. The nodes and collocation points are

$$
\xi_{k}=\cos \left(\frac{2 k-1}{2 n} \pi\right), \quad \eta_{j}=\cos \left(\frac{j}{n} \pi\right), \quad k=1 \ldots n, \quad j=1 \ldots n-1
$$

The quadratures are exact for $q_{k}$ interpolated by the polynomials of the $(2 n-1)^{\text {th }}$ degree. In the case when the RHS of (1) is a polynomial of $(2 n-2)^{\text {th }}$ order the solution of (3) represents the discrete analogue the exact solution of the system (1)-(2), see [2] for detail.

The stress intensity factors, SIF, at the right and left ends (designated by plus/minus respectively) are found by interpolation as follows

$$
K_{I}^{ \pm}-i K_{I I}^{ \pm}=\frac{1}{n} \sum_{k=1}^{n}(-1)^{k} q_{k} h_{k}^{ \pm}, \quad h_{k}^{+}=\cot \frac{2 k-1}{2 n} \frac{\pi}{2}, \quad h_{k}^{+} h_{k}^{-}=(-1)^{n}
$$

It is evident that the accuracy of the numerical solution is reduced if the RHS in (1) has discontinuities. In particular for concentrated loads the system (3) would produce trivial solutions. For step-like or piecewise loads the solution for a single rectilinear crack requires the use of a large number of nodes and collocation points to reach the accuracy within $0.1 \%$ (depending on the length of the loaded part).

For concentrated loads of intensity $P_{0}$ applied at the crack middle Savruk [1] suggested to seek solution as the sum of two functions

$$
g(t)=g_{1}(t)+g_{2}(t)
$$

These satisfy the following SIEs 


$$
\frac{1}{\pi} \int_{-1}^{1} \frac{g_{1}(t)}{t-x} d t=P_{0} \delta(x), \quad|x|<1
$$

where $\delta(x)$ is the Dirac delta function, and

$$
\begin{gathered}
\int_{-1}^{1}\left[\left(\frac{1}{t-x}+K(t, x)\right) g_{2}(t)+L(t, x) \overline{g_{2}(t)}\right] d t=f(x), \quad|x|<1 \\
f(x)=-\int_{-1}^{1}\left[K(t, x) g_{1}(t)+L(t, x) \overline{g_{1}(t)}\right] d t
\end{gathered}
$$

The advantage of this decomposition is that the latter SIE has a continuous RHS that can be approximated by a polynomial while the solution of the former SIE is expressed in the analytical form.

In this study we suggest to use a somewhat similar decomposition in application for the step-like loads, i.e. to replace SIE (8) by the following SIE

$$
\frac{1}{\pi} \int_{-1}^{1} \frac{g_{1}(t)}{t-x} d t=p(x) H(x-a) H(b-x), \quad|x|<1, \quad-1<a<b<1
$$

where $p(x)$ is a differentiable function and $H(x)$ is the Heaviside step function.

Hereafter it is assumed that half-length of the crack is unity.

The solution of (10) unbounded at both ends can be expressed in analytical form via the formula

$$
g_{1}(t)=\frac{-1}{\pi \sqrt{1-t^{2}}} \int_{a}^{b} \frac{\sqrt{1-x^{2}}}{x-t} p(x) d x+\frac{C}{\pi \sqrt{1-t^{2}}}, \quad|t|<1
$$

where, without loss of generality, one can assume $C=0$, which satisfies the condition in (2). This solution substituted into the second line of (9) determines the smooth RHS of (9) which is used further on to compute a numerical solution for $g_{2}(x)$ by the standard numerical scheme resulting in SLAE (3).

The stress intensity factors $K_{I}^{(1) \pm}-i K_{I I}^{(1) \pm}$ resulting from (10) are found by the following integral

$$
K_{I}^{(1) \pm}-i K_{I I}^{(1) \pm}=\frac{1}{\sqrt{\pi}} \int_{a}^{b} \frac{1 \pm x}{\sqrt{1-x^{2}}} p(x) d x
$$

while the SIFs $K_{I}^{(2) \pm}-i K_{I I}^{(2) \pm}$ resulting from SIE (9) are calculated by formula (6). Finally the total stress intensity factors is found as the sum 


$$
K_{I}^{ \pm}-i K_{I I}^{ \pm}=\left(K_{I}^{(1) \pm}+K_{I}^{(2) \pm}\right)-i\left(K_{I I}^{(1) \pm}+K_{I I}^{(2) \pm}\right)
$$

In the special case $p(x)=p_{0}=$ const the solution for $g_{1}(t)$ and the SIFs corresponding to this solution assume the form

$$
g_{1}(t)=\frac{-p_{0}}{\pi \sqrt{1-t^{2}}} \int_{a}^{b} \frac{\sqrt{1-x^{2}}}{x-t} d x=\frac{-p_{0}}{\pi \sqrt{1-t^{2}}}[F(t, b, 1)-F(t, a, 1)], \quad|t|<1
$$

where

$$
\begin{aligned}
& F(t, x, L)=\int \frac{\sqrt{L^{2}-x^{2}}}{x-t} d x=\sqrt{L^{2}-x^{2}}-t \arcsin \left(\frac{x}{L}\right)- \\
&-\sqrt{L^{2}-t^{2}} \ln \left(2 \frac{\sqrt{L^{2}-x^{2}} \sqrt{L^{2}-t^{2}}-t x+L^{2}}{x-t}\right)
\end{aligned}
$$

The stress intensity factors for a single crack loaded by the constant step-like load on the interval $(a, b)$ are calculated by the following formula

$$
\begin{aligned}
K_{I}^{(1) \pm}-i K_{I I}^{(1) \pm} & =\frac{p_{0}}{\sqrt{\pi}} \int_{a}^{b} \frac{1 \pm x}{\sqrt{1-x^{2}}} d x= \\
& =\frac{p_{0}}{\sqrt{\pi}}\left(\arcsin (b)-\arcsin (a) \mp \sqrt{1-b^{2}} \pm \sqrt{1-a^{2}}\right)
\end{aligned}
$$

The accuracy of the solution is examined in the following section for one special case $a=-b$ for which the SIFs in (16) assume the form

$$
K_{I}^{\text {step-like }}=\frac{2 p_{0}}{\sqrt{\pi}} \arcsin (b)
$$

This SIF will be use further on for comparisons and normalisation.

\section{Validation}

\subsection{Benchmark problem}

We further consider a benchmark SIE as follows

$$
\frac{1}{\pi} \int_{-1}^{1}\left(\frac{1}{t-x}+K_{1}(t) K_{2}(x)\right) g(t) d t=p_{0} H\left(b^{2}-x^{2}\right), \quad|x|<1, \quad 0<b<1
$$

where the kernel $L(x, t)=0$ and the kernel $K(t, x)=K_{1}(t) K_{2}(x)$ is degenerated and $p_{0}=$ const. 
The solution of (18) can be found as follows.

Firstly let us denote

$$
C_{1}=\frac{1}{\pi} \int_{-1}^{1} K_{1}(t) g(t) d t
$$

and rewrite (18) in the form

$$
\frac{1}{\pi} \int_{-1}^{1} \frac{g(t)}{t-x} d t=p_{0} H\left(b^{2}-x^{2}\right)-C_{1} K_{2}(x), \quad|x|<1, \quad 0<b<1
$$

An unbounded solution of (20) satisfying (2) can be presented in the form

$$
g(t)=\frac{-p_{0}}{\pi \sqrt{1-t^{2}}} \int_{-b}^{b} \frac{\sqrt{1-x^{2}}}{x-t} d x+\frac{C_{1}}{\pi \sqrt{1-t^{2}}} \int_{-1}^{1} \frac{\sqrt{1-x^{2}}}{x-t} K_{2}(x) d x, \quad|t|<1
$$

The former integral in the RHS of (21) is evaluated in the form

$$
\begin{aligned}
\int \frac{\sqrt{L^{2}-x^{2}}}{x-t} d x & =\sqrt{L^{2}-x^{2}}-t \arcsin \left(\frac{x}{L}\right)- \\
& -\sqrt{L^{2}-t^{2}} \ln \left(2 \frac{\sqrt{L^{2}-x^{2}} \sqrt{L^{2}-t^{2}}-t x+L^{2}}{x-t}\right)
\end{aligned}
$$

By denoting

$$
p_{2}(t)=\int_{-1}^{1} \frac{\sqrt{1-x^{2}}}{x-t} K_{2}(x) d x, \quad|t|<1
$$

one can present the solution in the form $(|t|<1)$

$$
g(t)=\frac{2 p_{0} t \arcsin (b)+C_{1} p_{2}(t)}{\pi \sqrt{1-t^{2}}}-\frac{p_{0}}{\pi} \ln \left(\left|\frac{b+t}{b-t}\right| \frac{\sqrt{1-b^{2}} \sqrt{1-t^{2}}+b t+1}{\sqrt{1-b^{2}} \sqrt{1-t^{2}}-b t+1}\right)
$$

The constant $C_{1}$ is found by multiplication of (9) by $K_{1}(t) / \pi$ followed by integration

$$
C_{1}=\frac{p_{0}}{D} \int_{-1}^{1}\left[\frac{2 t}{\sqrt{1-t^{2}}} \arcsin (b)-\ln \left(\left|\frac{b+t}{b-t}\right| \frac{\sqrt{1-b^{2}} \sqrt{1-t^{2}}+b t+1}{\sqrt{1-b^{2}} \sqrt{1-t^{2}}-b t+1}\right)\right] K_{1}(t) d t
$$

where 


$$
D=\pi^{2}-\int_{-1}^{1} \frac{p_{2}(t) K_{1}(t)}{\sqrt{1-t^{2}}} d t
$$

The stress intensity factors are found by limiting transition by letting $t$ tend to the ends (denoted as "+" for the right end and "-" for the left one)

$$
\begin{aligned}
K_{I}^{ \pm} & =\frac{1}{\sqrt{\pi}} \int_{-1}^{1} \frac{1 \pm x}{\sqrt{1-x^{2}}}\left(p_{0} H\left(b^{2}-x^{2}\right)-C_{1} K_{2}(x)\right) d x= \\
& =\frac{p_{0}}{\sqrt{\pi}}\left(2 \arcsin (b) \pm \frac{C_{1}}{p_{0}} p_{2}( \pm 1)\right)
\end{aligned}
$$

For example, let us assume

$$
K_{1}(t)=t, \quad K_{2}(x)=x
$$

Then

$$
p_{2}(t)=\int_{-1}^{1} \frac{x \sqrt{1-x^{2}}}{x-t} d x=-\pi\left(t^{2}-\frac{1}{2}\right)
$$

and (due to an odd integrand)

$$
\int_{-1}^{1} \frac{p_{2}(t) K_{1}(t)}{\sqrt{1-t^{2}}} d t=0
$$

Hence

$$
C_{1}=\frac{p_{0}}{\pi}\left(b \sqrt{1-b^{2}}+\arcsin (b)\right)
$$

The SIFs become

$$
K_{I}^{ \pm}=\frac{p_{0}}{\sqrt{\pi}}\left(2 \arcsin (b) \mp \frac{1}{2}\left(b \sqrt{1-b^{2}}+\arcsin (b)\right)\right)
$$

In this case the accuracy of the numerical solution for the SIFs is mainly defined by the accuracy of the calculation of the integral for $C_{1}$. For the tolerance of $10^{-5}$ in software MATHCAD 6+ used for calculation of the integral in (10) with the integrand with the logarithmic function and $n=4$ the numerical solution is accurate within 6 significant digits for $10^{-4}<b<1$. 


\subsection{Two parallel cracks}

Let us compute the SIFs for the case of two parallel cracks of the same size subjected to the same step-like loads of unit intensity $p_{0}=1$. This configuration is shown in Fig. 1, it is characterised by is the distance, $d$, between the crack centres, the angle, $\beta$, between the line connecting the crack centres and the cracks and the size of the loaded part $2 b$.

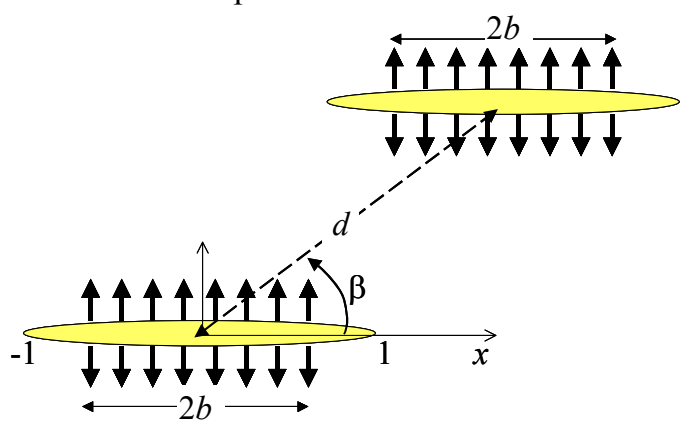

Figure 1: Two parallel shifted cracks.

In this case the kernels can be derived from the form presented in [1] as follows

$$
K(t, x)=\operatorname{Re} \frac{1}{t+x-d e^{2 i \beta}}, \quad L(t, x)=\frac{i d \sin (2 \beta)}{\left(t+x-d e^{-2 i \beta}\right)^{2}}
$$

For $\beta=0$ the cracks are collinear and for $\beta=\pi / 2$ they are parallel as shown in Fig. 2.
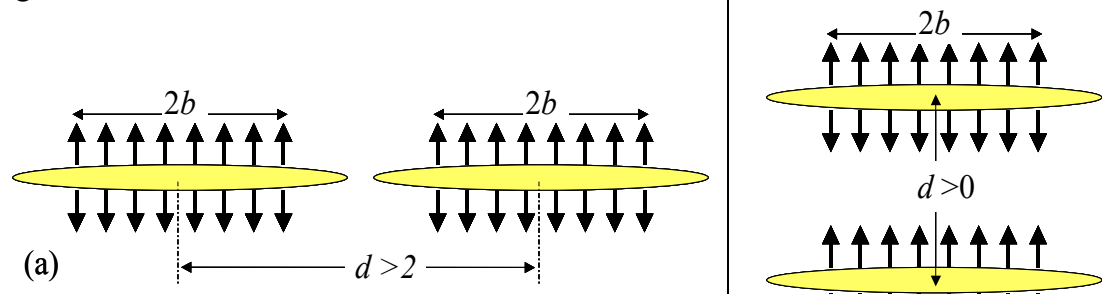

$d>0$

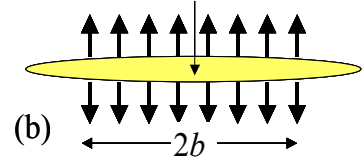

Figure 2: Collinear and parallel cracks under step=like loads.

Calculations have been performed for two configurations presented in Fig. 2. Verifications have been performed for the case $b=1$ (fully loaded crack) to compare the results with those presented in [1] and [3]. The results of calculations are summarised in Table 1 for the case of collinear cracks (Fig. 2(a) and (b) and in Table 2 for the case of parallel cracks (Fig. 2(b)). The data in the tables show SIFs are normalised by the stress intensity factor for a crack loaded 
by a constant normal load $p_{0}$, i.e. by $K_{\mathrm{I}}=p_{0}(\pi l)^{1 / 2}$, where the crack half-length $l=1$.

Table 1 presents $K_{\mathrm{I}}$ for the inner and outer ends of the collinear cracks in the form $\left(\mathrm{K}_{\mathrm{I}}^{\text {inner }}, K_{\mathrm{I}}{ }^{\text {otter }}\right)$. Table 2 shows SIFs for parallel cracks in the complex form $K_{\mathrm{I}}+i K_{\mathrm{II}}$. The first rows in both tables correspond to the case $b=1$, these results coincide with the known solutions presented in [1] and handbook [3].

Graphical representation of the results of the calculated mode I SIFs are shown in Fig. 3 for collinear cracks (inner ends) and in Fig. 4 for parallel cracks. In both figures the SIFs are normalised by the mode I stress intensity factor for a single crack under step-like load given by formula (17). It can be seen that the collinear cracks always magnify the SIFs, while the parallel cracks exhibit, so called, shielding effect, i.e. the SIFs are smaller than the SIF in (17) for the same loads.

Table 1: $\quad$ SIFs for collinear cracks under step-like loads for different $b$ and $d$. First data for the inner end, second data for the outer end.

\begin{tabular}{|c|c|c|c|c|c|}
\hline$b \backslash d$ & 2.1 & 2.25 & 2.5 & 3.0 & 4.0 \\
\hline 1.00 & $1.795,1.151$ & $1.414,1.112$ & $1.229,1.081$ & $1.112,1.052$ & $1.048,1.028$ \\
\hline 0.95 & $1.569,0.946$ & $1.203,0.908$ & $1.023,0.878$ & $0.909,0.849$ & $0.845,0.825$ \\
\hline 0.90 & $1.449,0.856$ & $1.103,0.820$ & $0.931,0.791$ & $0.821,0.762$ & $0.759,0.740$ \\
\hline 0.75 & $1.156,0.664$ & $0.873,0.633$ & $0.729,0.608$ & $0.634,0.584$ & $0.581,0.564$ \\
\hline 0.50 & $0.741,0.419$ & $0.559,0.398$ & $0.463,0.381$ & $0.399,0.364$ & $0.362,0.350$ \\
\hline 0.25 & $0.364,0.204$ & $0.274,0.194$ & $0.227,0.185$ & $0.195,0.177$ & $0.176,0.170$ \\
\hline 0.10 & $0.145,0.081$ & $0.109,0.077$ & $0.090,0.074$ & $0.077,0.070$ & $0.070,0.067$ \\
\hline 0.05 & $0.072,0.041$ & $0.054,0.039$ & $0.045,0.037$ & $0.039,0.035$ & $0.035,0.034$ \\
\hline
\end{tabular}

Table 2: $\quad$ SIFs for parallel cracks under step-like loads for different $b$ and $d$.

\begin{tabular}{|c|c|c|c|c|c|}
\hline$b \backslash d$ & 0.4 & 0.8 & 1.0 & 2.0 & 4.0 \\
\hline 1.00 & $0.721+0.163 \mathrm{i}$ & $0.758+0.13 \mathrm{i}$ & $0.773+0.117 \mathrm{i}$ & $0.843+0.061 \mathrm{i}$ & $0.930+0.016 \mathrm{i}$ \\
\hline 0.95 & $0.527+0.163 \mathrm{i}$ & $0.560+0.130 \mathrm{i}$ & $0.575+0.116 \mathrm{i}$ & $0.643+0.061 \mathrm{i}$ & $0.729+0.016 \mathrm{i}$ \\
\hline 0.90 & $0.456+0.161 \mathrm{i}$ & $0.482+0.128 \mathrm{i}$ & $0.496+0.114 \mathrm{i}$ & $0.561+0.060 \mathrm{i}$ & $0.645+0.016 \mathrm{i}$ \\
\hline 0.75 & $0.336+0.143 \mathrm{i}$ & $0.343+0.119 \mathrm{i}$ & $0.351+0.106 \mathrm{i}$ & $0.404+0.054 \mathrm{i}$ & $0.479+0.014 \mathrm{i}$ \\
\hline 0.50 & $0.208+0.097 \mathrm{i}$ & $0.204+0.088 \mathrm{i}$ & $0.205+0.080 \mathrm{i}$ & $0.236+0.041 \mathrm{i}$ & $0.290+0.010 \mathrm{i}$ \\
\hline 0.25 & $0.101+0.048 \mathrm{i}$ & $0.098+0.046 \mathrm{i}$ & $0.097+0.042 \mathrm{i}$ & $0.110+0.022 \mathrm{i}$ & $0.138+0.005 \mathrm{i}$ \\
\hline 0.10 & $0.040+0.019 \mathrm{i}$ & $0.039+0.018 \mathrm{i}$ & $0.038+0.017 \mathrm{i}$ & $0.043+0.009 \mathrm{i}$ & $0.055+0.002 \mathrm{i}$ \\
\hline 0.05 & $0.020+0.010 \mathrm{i}$ & $0.019+0.009 \mathrm{i}$ & $0.019+0.009 \mathrm{i}$ & $0.022+0.004 \mathrm{i}$ & $0.027+0.001 \mathrm{i}$ \\
\hline
\end{tabular}




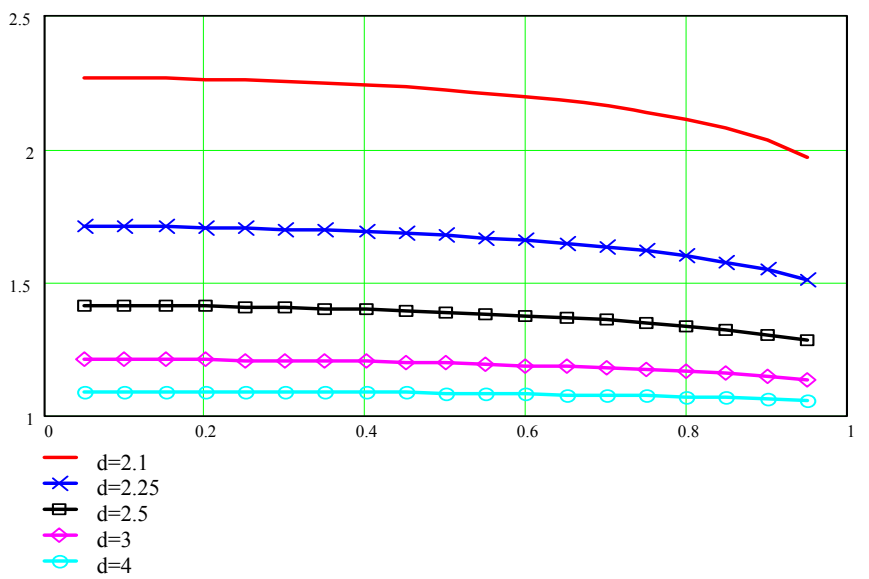

Figure 3: Dimensionless $K_{\mathrm{I}}$ for the inner ends of two collinear cracks vs. $b$ for different $d$.

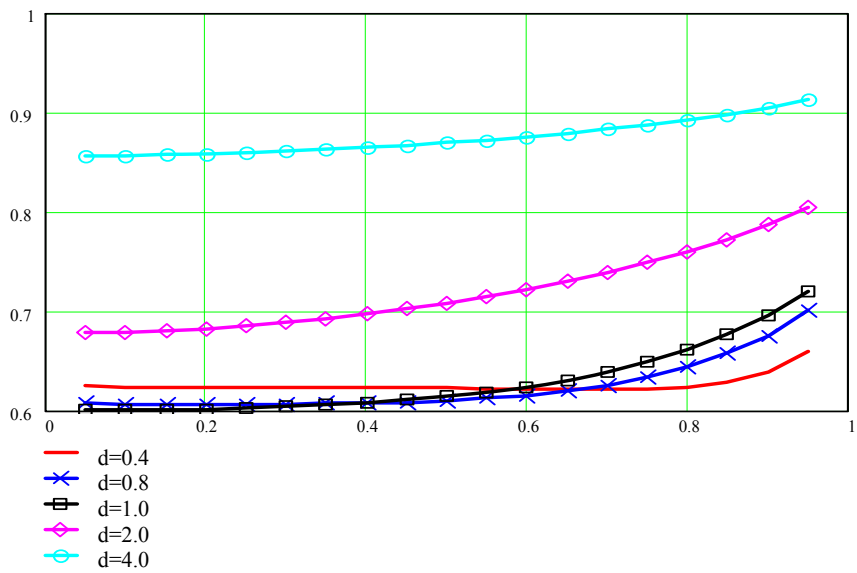

Figure 4: Dimensionless mode I stress intensity factor for two parallel cracks vs. $b$ for different $d$.

\section{Conclusions}

The paper presents an effective technique to numerical solutions of singular integral equations for plane crack systems subjected to discontinuous loads. Verifications performed for the benchmark equation specified in subsection 3.1 revealed effectiveness of the proposed approach. Applications for the case of two collinear cracks also agree with semi-analytical solutions in [3]. The solutions for parallel cracks reveal the existence of the shielding effect for the range of the 
parameters studied. The later results can be used for advanced design of multistage hydrofractures in elastic reservoirs.

\section{Acknowledgement}

The author is grateful to the Russian Foundation for Basic Research for the financial support of this work.

\section{References}

[1] Savruk P.M. Two-dimensional problems of elasticity for crack problems, Naukova Dumka, Kiev, 1981.

[2] Erdogan, F. and Gupta, G.D. 1972. On the numerical solution of singular integral equations, Quart. Appl. Math. 29, 525-534.

[3] Murakami Y. Stress Intensity Factors Handbook, Pergamon, 1987. 